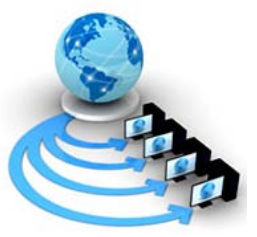

Volume 9, No. 2, March-April 2018

RESEARCH PAPER

Available Online at www.ijarcs.info

\title{
ENERGY EFFICIENT DISTRIBUTED CLUSTERING AND SCHEDULING ALGORITHM FOR WIRELESS SENSOR NETWORKS WITH NON-UNIFORM NODE DISTRIBUTION
}

\author{
Mumtaz Ahmed \\ Department of Computer Engineering, \\ Jamia Millia Islamia \\ New Delhi, India
}

\author{
M. N. Doja \\ Department of Computer Engineering, \\ Jamia Millia Islamia \\ New Delhi, India
}

\author{
Mohd Amjad \\ Department of Computer Engineering, \\ Jamia Millia Islamia \\ New Delhi, India
}

\begin{abstract}
The lifetime of Randomly Distributed wireless sensor networks gets effected do to the imbalanced energy consumption in sensor nodes. The energy consumption is balanced among sensor nodes by using the efficient clustering algorithm that is proposed in this research paper. there are two cluster phases of EECS one is setup phase and another is steady state phase. The cluster election algorithm selects cluster head which uses sensor nodes local information. In the steady state phase, the time slots are allotted for member nodes as per the data which is available in sensor nodes. As compared to SA-EADC and EADC, the simulation results of EECS is better when viewed in consumption of energy and lifetime of a network
\end{abstract}

Keywords: Clustering. Scheduling, Energy Efficient, Slot Allotment Algorithm, TDMA, Wireless Sensor Networks.

\section{INTRODUCTION}

Wireless sensor networks [1] (WSN) are the combination of small sensing self-powered sensors that are capable of operating in harsh conditions. Sensors are used to monitor the activities of interest in particular sensing field and collected data is communicated to the base station of the wireless medium. The data is then computed and clubbed together to get the desired result. Sensors can be deployed depending on the type of area if the area is known or sensing field knowledge is known in advance, then we can deploy sensors in a planned manner otherwise, if there is no prior knowledge of sensing field then we need to deploy sensors randomly. WSN finds a large number of important applications like in environment monitoring, battle filed control, healthcare,and weather forecasting. The complex arbitrary nature of clustering protocol[5] makes its design difficult for wireless sensor network technology. The clustering protocol should be designed in such a way that the consumption of energy should be reduced or minimized and also the data communication reliability should be maintained. The cluster nodes transmit the data as packets to the cluster head. The cluster head then fuses the data and forwards it to the sink. The decrease in a number of transmissions reduces the energy consumption and minimizes the data packet redundancy, and thus the bandwidth resources[2][3] are saved.

In cluster sensor network, the energy consumption in sensors defines the lifetime of the network. So if the energy consumption is not balanced, the network lifetime is decreased. Similarly, the network lifetime is increased by distributing uniformly the cluster heads, which in turn balances the energy consumption among the nodes.
The time slots are allotted to membership nodes usingTDMA[6] scheduling, and the cluster heads broadcast the TDMA schedule packets which contain node slot number and membership. In each defined time slot, one sensor

transmits data. The nodes communicate directly through a cluster head.

The best way to allocate slots to member nodes is that cluster head allocates dynamically on demand of member nodes rather than using uniform slot scheduling. If a node is not allotted a time slot, then the node goes into sleep mode in current session and wats for next session to request cluster head for allocating a time slot to transmit data.

Prediction-based energy Efficient Distributed Clustering for WSNthat supports non-uniform node distribution scheduling algorithm is proposed. There are 2 phases of EECS, the cluster setup phase,andsteady-statephase. Local information of nodes is used to decide cluster head by using a newly designed algorithm. For scheduling of time slots, we use Round Robbin to allocate the time slots in steady-state phase. In the end, we analyze simulation results generated in NS2 and compare those results with existing clustering algorithms.

\section{RELATED WORK}

In designing a wireless sensor network, the energy consumption is the key issue. For WSN, there are various energy efficient clustering protocols. One clustering based routing protocol for WSN suggested by Yu.et.al [8] which is based on energy-aware clustering algorithm which supports non-uniform node distribution. There is also another algorithm called scheduled activity energy aware distributed clustering [9] (SA-EADC) based on EADC that exploits the 
redundant sensor and turn them off for the current round. The redundant nodes are scheduled to work on residual energy. To minimize energy consumption "A Local Energy Consumption Prediction Based Clustering Protocol for Wireless Sensor Networks" [10] (LECP-CP) is proposed by taking more accurate and realistic cluster radius into consideration and a new cluster head election mechanism is defined on the basis of local energy consumption of node. T. H. Hsu.et.al [6] proposed “Adaptive Time Division Multiple Access Based Medium Access Control Protocol for Energy Conserving and Data Transmission in Wireless Sensor Networks" based on TDMA-based MAC protocol to save energy and increases data transmission efficiency of nodes in the cluster-based WSN networks.

"Unequal cluster-based routing protocol" is another protocol in WSN that was proposed by G. Chen. Et.al [11]. J.Ma.et.al [12] proposed "Energy Efficient TDMA Sleep Scheduling in WSN". "Energy-Efficient Prediction Clustering Algorithm Multilevel Heterogeneous Wireless Sensor Network” given by Jian Peng.et.al [13] and various other protocols.

\section{NETWORK MODEL}

In the $M \times M$ squaring field we need to deploy $\mathrm{n}$ sensors. The following assumptions are taken into consideration while describing the network model.

1. The sensor nodes and base station nodes should be stationary.

2. In a sensing field, there is n number of sensors to beplaced uniformly.

3. In terms of energy and location sensors must be heterogeneous.

4. The data is forwarded to the base station via cluster head in continuous form.

5. Each sensor uses power control process to vary node transmission power.

Radio dissipation model [7] is used for the analysis of energy consumption in all sensor nodes. This model states that in the transmission of the node, the energy consumption depends upon the summation of constant electronic components energy and amplifier energy which is proportional to receiver distance. .In order to transmit a messagetol bitsthe consumed energy $E_{T x}(l, d)$ by radio is given by

$E_{T x}(l, d)=\left\{\begin{array}{l}l\left(\varphi+\alpha d_{(i, j)}^{2}\right) d_{(i, j)} \leq d_{0} \\ l\left(\varphi+\beta d_{(i, j)}^{4}\right) d_{(i, j)}>d_{0},\end{array}\right.$

where $\varphi$ is energy by electronic components is the onstant, $\alpha$ and $\beta$ amount of energy dissipated in transmitter amplifier per bit. $d_{(i, j)}$ is the distance between nodes i.e. node $\mathrm{i}$ and $\mathrm{j}$. The energy consumed in bit message is given by

$$
E_{R x}(l)=l \times \varphi
$$

\section{EECS ALGORITHM}

In this section, we explain the EECS in detail. The algorithm is divided into two phases, the first phase is called as cluster setup phase and the second phase is known as steady set up phase.

Cluster setup phase is further divided into four phases: local observation and data collecting phase, cluster head phase, activation phase and cluster formation phase. The steady phase has a sub phase called as transmission phase that consist $\mathrm{k}$ sessions with each session having a fixed duration. In every session, we have contention, data transmission and idle periods. We assume that for mnon- cluster head node communicationwe requiremslots. The TDMA slot is not allotted in current round for cluster head nodes that doesn't have any data to transmit, but nodes having data to transmit are given more time slots. The nodes go into sleep mode when there is no data to transmit thus saving energy. On the basis of local observation function cluster nodes are elected. The local observation function is the mean of average residual energy and average energy consumption of neighboring sensor to residual energy and expected consumption of node itself.

\section{A. Cluster Setup Phase}

This phase is further subdivided into 5 sub-phases namely local observation, information gathering phase, cluster head phase, cluster head competition phase, sensor redundancy and cluster formation phase. The duration of each phase is $\mathrm{T} 1, \mathrm{~T} 2$, $\mathrm{T} 3$, T4, and T5 respectively. The slot allotment algorithm is implemented in Steady State Phase. The table below shows various types of msgs used.

Table 1Description of Control Message in EECS

\begin{tabular}{|l|l|}
\hline .Node_Msg & Tuple(self_id, self-energy) \\
\hline Head_Msg & Tuple(self_id) \\
\hline Join_Msg & Tuple(self_id, head_id) \\
\hline Schedule_Msg & Tuple(sechdule_order) \\
\hline Sleep_Msg & Tuple(self_id, self_status) \\
\hline
\end{tabular}

\section{Local observation and information gathering phase.}

In this phase,the Node_Msg is broadcasted by each sensor to its neighboring sensor node that lies within its transmission range $\mathrm{r}$ and each message contains two fields: sensor id and current energy level of the sensor. Each sensor node receives Node_Msg simultaneously from its neighbor nodes. In order to send messages to its neighboring nodes, each node $s_{i}$ calculates the athematic mean of residual energy $E_{i a}$ and also the arithmetic mean of consumption of energy $E_{i a c}$ of its neighbor node. Each sensor node $s_{i}$ defines observation $O$ (I) from its neighbouring sensors. The observation function $O($ II) is given by equation:

(i) $=\frac{E_{i a c}}{E_{i c}} * \frac{E_{i a}}{E_{\text {ir }}}$

Where $E_{i r}$ and $E_{i c}$ is residual energy and energy consumption of the node $s_{i}$ respectively. The waiting time $t_{i}$ to broadcast Head_Msg is given by: 
$t_{i}=o(i) * T_{2} * V_{r}$

T2 is time duration, $\mathrm{Vr}$ is real value uniformly distributed between [0.9-1] that is used to minimize the probability of broadcasting Head_Msg by more than one node simultaneously.

The cluster phase flowchart is shown below:

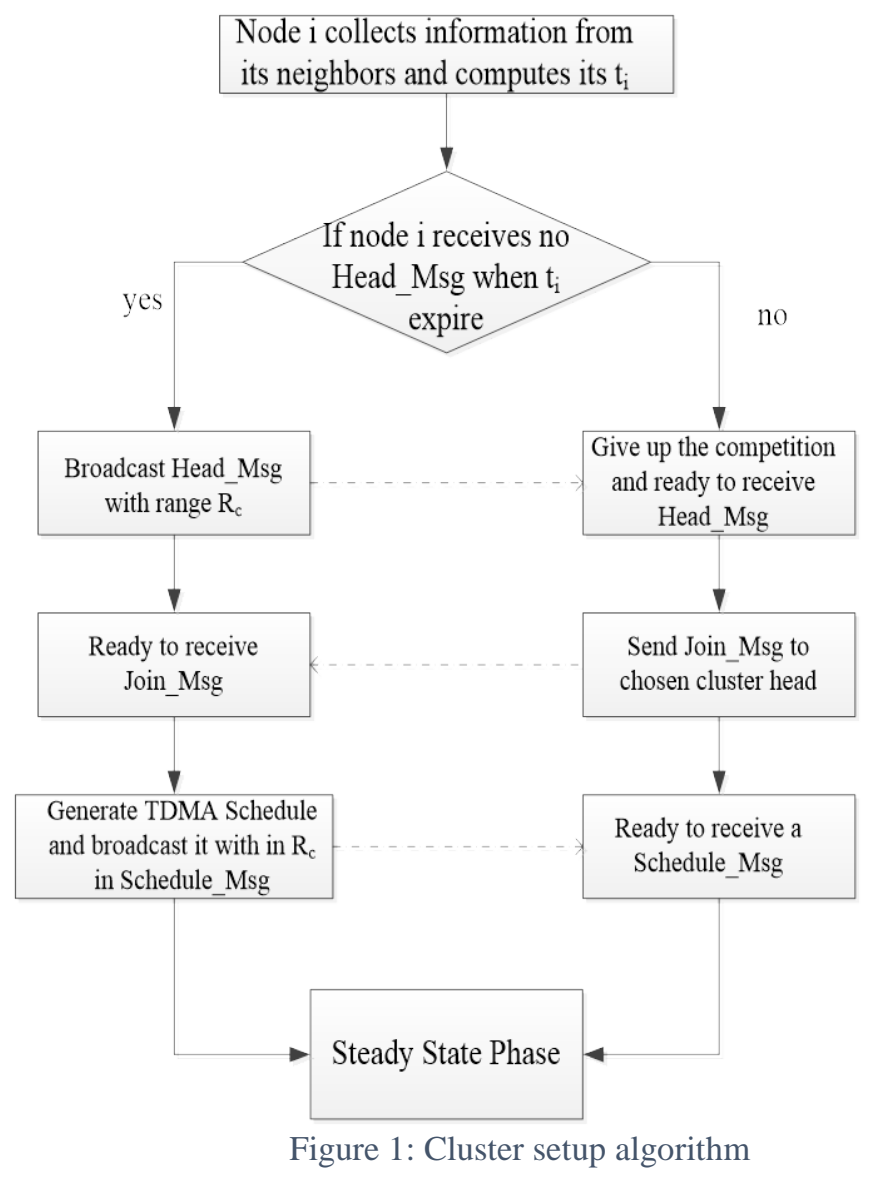

\section{Competition phase in the cluster head}

The phase is started when the time duration $T_{2}$ expires in EECS algorithm. During this time, the node itself broadcast the Head_msg to its neighboring nodes in range $R_{c}$, if it doesn't receive any Head_msg. if the Head_msg is not sent by a node, the sensor node becomes a plain node. when the Head_Msg is broadcasted by node $s_{i}$ then node waits $2 *$ for for for $\Delta T$ time so that there exists other head Head_Msg broadcasted by neighboring nodes in its range Rc, $\Delta T$ denotes the time interval that guarantee that all nodes in cluster range Rc receive Head_Msg but if $s_{i}$ has does not receive any Head_Msg in time duration $\Delta T$ then it will set its state as Head.

\section{Activation and Redundancy Check Phase}

In this phase, the coverage redundancy and activity of plain nodes are properly checked. Each sensor knows their status whether it isaplain node or a cluster head. The cross coverage[14] checks are executed to eliminate redundancy. In each round, the node is highly active if it has high energy.The timer of the redundant sensor is set proportional to its residual energy. If no Sleep_Msg is transmitted to the redundant node during its expiration time, then a Sleep_Msg is broadcasted by a redundant node in a range of $2 \mathrm{Rc}$ and status is set to inactive and node goes to sleep mode for the current round. Otherwise, the active direct neighboring list generated and redundancy check is defined again. The algorithm used in phase 1 is given below:

\section{Algorithm 1: Cluster Setup Algorithm}

Begin: cluster setup algorithm cluster_state $<$ - Candidate current_status $<$ - Active

/* Check T_1 if not Expired */ while(True) do if(!T_1.Expire()) then

Receives the Msg from Node

Neighbourhood sensor table is updated NSTT [ ] active direct neighbor sensor table is updated ADNSTT [ ] endif if(T_1. Expire()) then

Exit ()

End endif

$$
t_{-} i \leftarrow \text { broadcast_Busy_Time }
$$

/* Check T_2 if not Expired */ while(True) do

if(!T_2.Expire()) Begin

if $\left(\right.$ Current_Time $\left.\leftarrow t_{-} i\right)$ then

Begin

if(Receive a Head_Msg from a neighbor sensor NSTT[j] do cluster_state $\leftarrow$ plain NSTT $[j]$.cluster_state $\leftarrow$ Head

else

continue

endif

else if(cluster_state $=$ Candidate) then cluster_state $\leftarrow$ Head

Broadcast Head_Msg wait $2 * \Delta \mathrm{t}$

endif

endif

if (T_2. Expire ()) Exit ()

End

$$
\begin{aligned}
& \text { /* Check T_3 if not Expired */ } \\
& \text { While (True) do }
\end{aligned}
$$

if (! T_3. Expire ()) Then

if (cluster_state=plain and location!=boundary) then cross coverage algorithm is executed while(Redundant()=True) do

Set Timer $\alpha$ E_residual

Wait till timer expires

if( Sleep_Msg is received from an active direct neighbor node) then Update ADNSTT[ ]

Execute cross coverage algorithm else

Broadcast Sleep_Msg

current_status $\leftarrow$ Inactive 


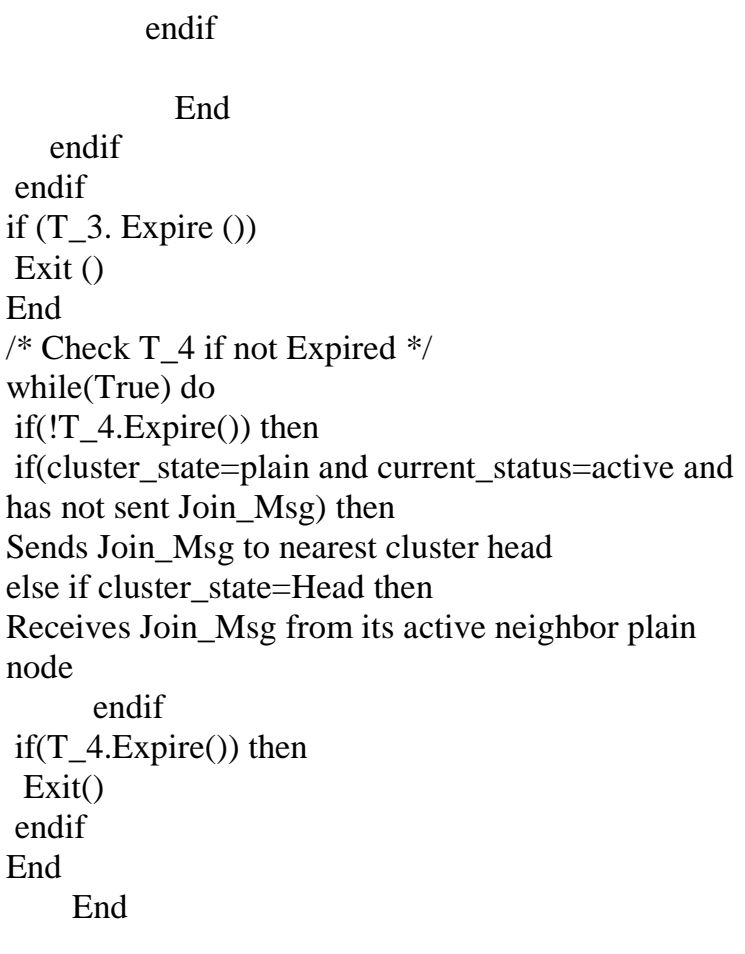

\section{Cluster Phase Formation}

This is the last phase that starts at time duration $\mathrm{T}_{4}$. For a node,a cluster head is chosen that is closest to it which is based on strength of the signal. The plain nodes simply send a Join_Msg. The time of data transmission for the nodes is calculated by a Schedule_Msg (TDMA Schedule) to all plain nodes within range Rc. The cluster set up phase completes only once the TDMA schedule is known to plain nodes.

\section{B. Steady State Phase}

This phase has many sessions like Contention Period, Data Transmission, Advertisement period and Idle Period in each session. The data transmission period may vary but Data Plus Idle period is fixed. Every node gets active in contention period (CP) and it follows TDMA schedule. Each node can transmit 20 Byte control messageonly in a given specific time slot if it has data otherwise slot remains empty.

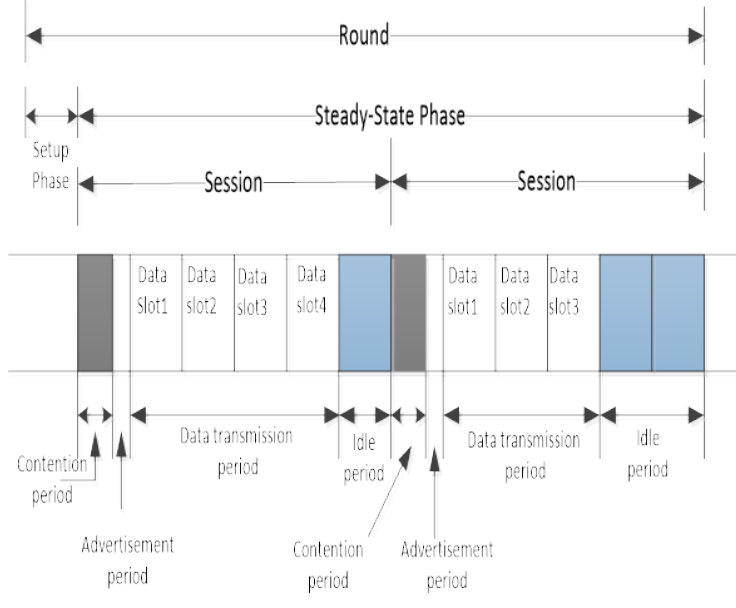

Fig. 2: Design of a single round

The Round Robin method is used to implement transmission schedule. Sensors with more observed data can request Cluster Head for more time slots for data transmission. Otherwise, if a sensor node has no data then it can go into sleep mode in order to save energy and more time slots are allocated to nodes having data. The algorithm that runs on Cluster Head for time slot allocation based on Round Robin is given below:

\section{Algorithm 2: Slot Allotment Algorithm}

begin (slot allotment algorithm) require slot Array: represent the require slot of sensor (index: sensorid, content: required slot value) allot slot Array: represent the alloted slot of sensor (index: slotid, content: sensorid)

$$
\begin{gathered}
\text { while(T5 not expired) do } \\
k=1 ; \\
\mathrm{x}=\operatorname{Max}(\text { requires slotArray) } \\
\text { For } \mathrm{j}=1 \text { to } \mathrm{x} \text { do } \\
\text { For }(\mathrm{i}=1 \text { to last index of require slot) do } \\
\text { if (required slot Array }[\mathrm{i}] \neq 0 \text { and available slot }>0 \\
\text { and state }=\text { plain and status = active ) } \\
\text { allot slot Array }[\mathrm{k}]=\mathrm{i} ; \\
\text { required slotArray }[\mathrm{i}]=\text { required slot Array }[\mathrm{i}]-1 \text {; } \\
\text { increment } \mathrm{k} \text { by } 1 \text {; } \\
\text { decement avalable slot by } 1 ; \\
\text { endif } \\
\text { endfor } \\
\text { end }
\end{gathered}
$$

We assume the number of slots is required equal to a number of plain nodes cluster. The slots are allocated based on RR scheduling. Suppose if a node S1, S2, and S10 need two slots, node S3, S4, S6, S7and S9 have no data to transfer, S5 needs one-time slots and S8 needs three-time slots. This scenario is shown below: 


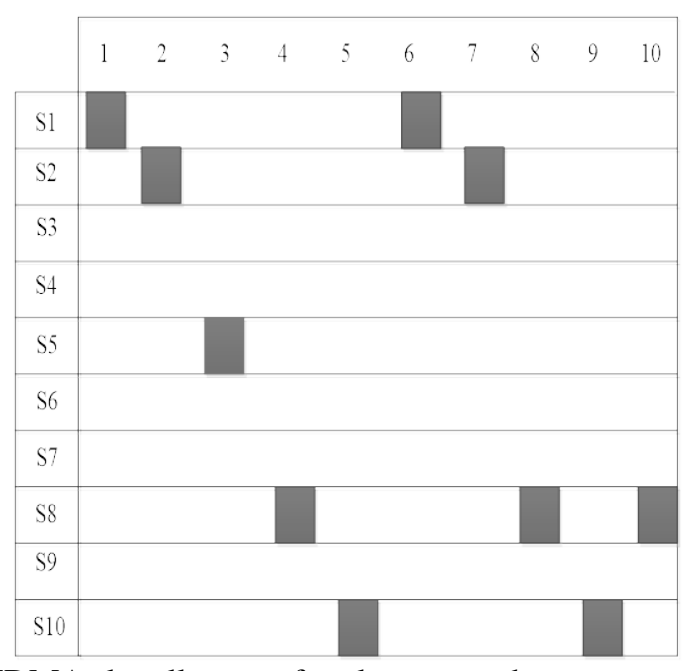

Fig. 3. TDMA slot allotment for cluster member sensor

\section{RESULTS AND DISCUSSION}

The performance analysis of proposed EECS is done in NS2and is presented in this section. Theperformance of proposed clusteringprotocolwith existing protocols. For validation of EECS performance, we implemented sensor networks in an area of $200 * 200 \mathrm{~m}^{2}$ and effect of signal collision and interference is neglected. EECS is implemented in two scenarios: Nodes are randomly distributed and in the second scenario, nodes are grouped together in certain part of the network. The simulation parameters are given in the table below:

Table 2: Simulation Parameters

\begin{tabular}{|l|l|}
\hline Number of & 100 \\
\hline Sink location & $(250,100)$ \\
\hline Initial energy of & $1-3 \mathrm{j}$ \\
\hline$\varphi$ & $50 \mathrm{nj} / \mathrm{bit}$ \\
\hline$\alpha$ & $10 / \mathrm{pj} / \mathrm{bit} / \mathrm{m} 2$ \\
\hline$\beta$ & $0.0013 \mathrm{pj} / \mathrm{bit} / \mathrm{m} 4$ \\
\hline Data Packet size & $5000 \mathrm{bits}$ \\
\hline Control packet & $500 \mathrm{bits}$ \\
\hline Sleep power & $15 \mu \mathrm{W}$ \\
\hline$E_{\text {sen }}$ & $0 \mathrm{j} / \mathrm{bit}$ \\
\hline$E_{\text {com }}$ & $5 \mathrm{nj} /(\mathrm{bit}$ signal) \\
\hline
\end{tabular}

\section{A. Network lifetime}

Network lifetime is expressed when entire network is operational. To evaluate the effect of sensing range on network lifetime we set $20 \mathrm{~m}$ and $25 \mathrm{~m}$ sensing range for both scenarios. The resultsobtained in our protocol show network lifetime per round is increased as compared to SA-EADC and EADC. We observed that if we increase sensing range in EECS the network lifetime also gets increased. The results show improvement by $7.5 \%$ and $11 \%$ in scenario 1 when sensing range is $20 \mathrm{~m}$ as compared to SA-EADC and EADC algorithms respectively. However, if we increase the range to $25 \mathrm{~m}$, the network lifetime increases $23 \%$ and $27 \%$ as compared to SA-EADC and EADC respectively.

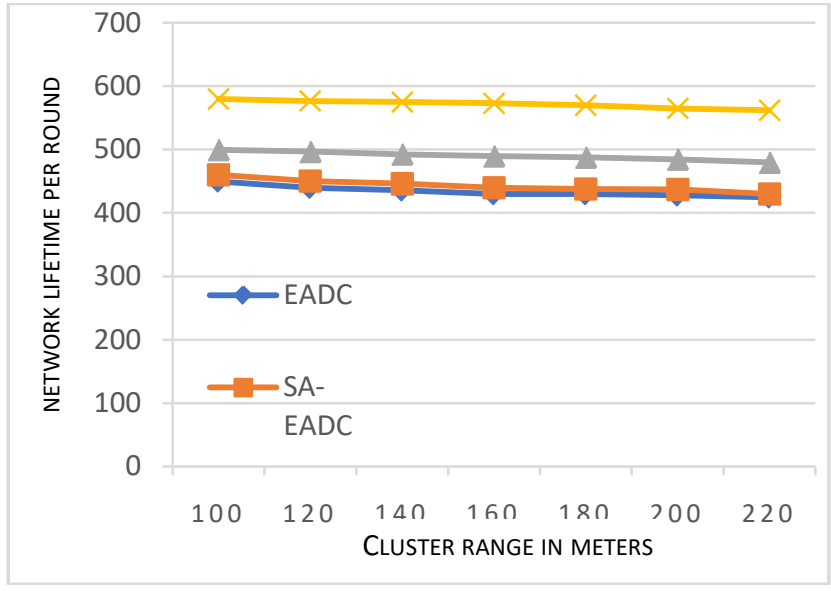

Fig. 4: Senario1 Network lifetime

In scenario 2, when the sensing range sets equal to $20 \mathrm{~m}$, network lifetime improved by $7.6 \%$ and $10.3 \%$ for the sensing range $20 \mathrm{~m}$ as compared with the SA-EADC and EADC algorithms respectively.

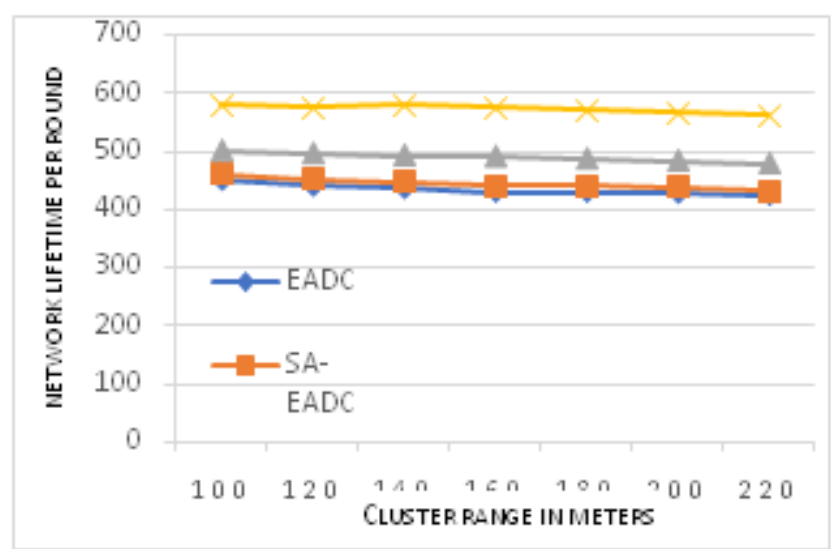

Fig. 5. Network lifetime for senario2

\section{B. Energy Consumption}

Energy consumption is defined as the average energy which is consumed by nodes consumption while topology construction, data transmission and sleep mode per rounds. The analysis shows network life is better in EECS as compared to EADC and SA-EADC respectively. 


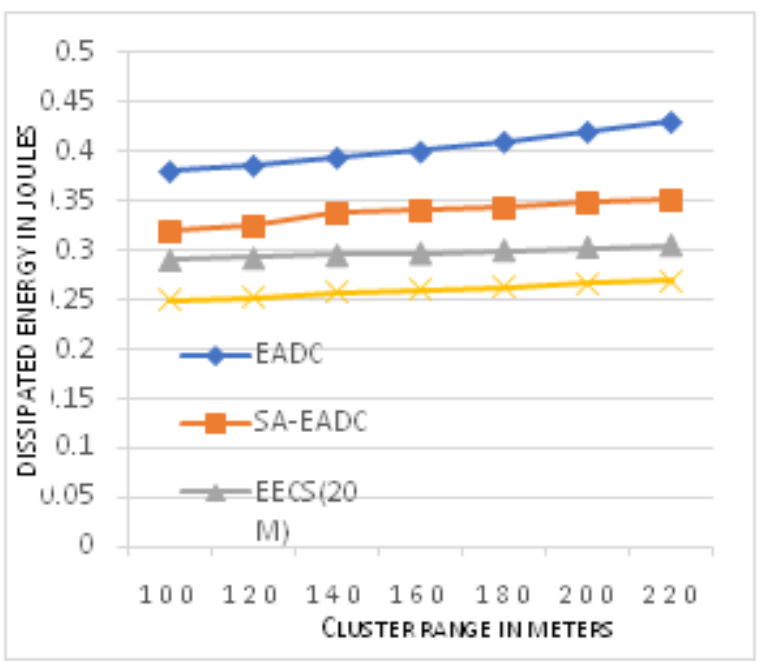

Fig. 6. Average energy dissipation per round for senario1

In second scenario energy consumption is decreased by $15 \%$ and $24 \%$ for $20 \mathrm{~m}$ range as compared to SA-EADC and EADC respectively.

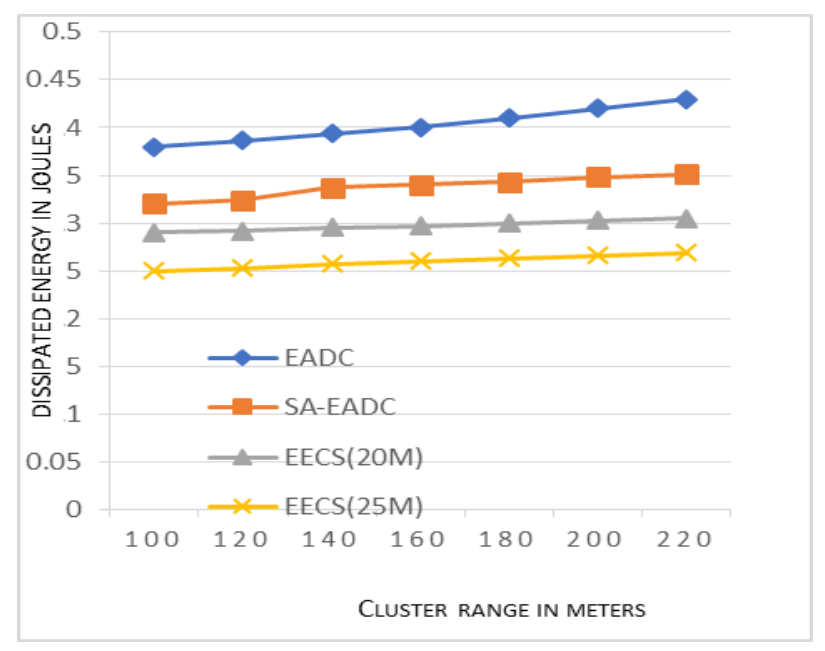

Fig. 7. Average energy dissipation per round for senario2

\section{CONCLUSION}

In general, from the last decade, there has been a good research in clustering of wireless sensor networks. In this proposed paper we particularly focused on main characteristics of wireless sensor networks like network lifetime and energy consumption. As it was observed that EECS paves way for clustering of WSNs. This protocol leads to energy efficient routing in WSNs. The scalability in WSNs is supported by using scheduling algorithm. Since it reduces communication overhead and energy consumption which is independent of network size, thus becomes suitable for realtimelarge-scale WSNs. In future,we will try to further maximize energy efficiency by choosing the optimal number of clusters. We will also try to develop recovery protocols needed in case the cluster head fails.

\section{REFERENCES}

[1]. I. Akyildz, W. Su, Y. Sankarasubramaniam, \& E. Cayirci, (2000) “A Survey on Sensor Network", IEEE Communication Magzine, Vol.40, No. 8, pp.102-114.

[2]. G. Anastasi, M. Conti, M. D. Francesco, A. Passarella, (2009) "Energy conservation in wireless sensor networks: A survey”, Ad Hoc Networks, Vol. 7, No. 3, pp. 537-568.

[3]. K. Akkaya, M. F. Younis, "A Survey on Routing Protocol for Wireless Sensor Networks",Ad Hoc network, Elsevier, Vol. 3, No. 3, 2005, pp. 325-349.

[4]. A. A. Abbasi, M. F. Younis, "A survey on clustering algorithms for wireless sensor networks", Computer Communications, Vol. 30, No. (14-15), 2007, pp. 28262841.

[5]. G. Chen, C. Li, M. Ye, J. Wu, “ An Unequal Cluster-Based Routing Protocol in Wireless Sensor Network” Wireless Networks, Springer, Vol. 15, No. 2, 2009, pp. 193-207.

[6]. T. H. Hsu, P. Y. Yen, “Adaptive Time Division Multiple Access Based Medium Access Control Protocol for Energy Conserving and Data Transmission in Wireless Sensor Networks,” IET Communication, Vol. 5, No. 18, 2011, pp. 2662-2672.

[7]. W. B Heinzelman, A. P. Chandrakasan, H. Balakrishnan, "An Application-Specific Protocol Architecture for Wireless Microsensor Networks," IEEE Transaction on Wireless Communication, Vol. 1, No. 4, 2002, pp. 660670.

[8]. J. Yu, Y. Qi, G. Wang \& X, Gu (2012) “A cluster-based routing protocol for wireless sensor networks with nonuniform node distribution", International Journal of Electronic and Communication, Elsevier, pp.54-61.

[9]. N. Nokhanji, Z. M. Hanapi, S. Subramaniam, M. A. Mohamed, A scheduled Activity Energy-Aware Distributed Clustering Algorithm for Wireless Sensor Networks with Nonuniform Node Distribution," International Journal of Distributed Sensor Networks, Hindawi, Vol. 2014, 2014, PP. 133-139.

[10]. J. Yu, L. Feng, L. Jia, X. Gu, D. Yu, “ A Local Energy Consumption Prediction Based Clustering Protocol For Wireless Sensor Networks,” Sensors, Vol. 14, No. 8, 2014, pp. 23017-23040.

[11]. G. Chen, C. Li, M. Ye, J. Wu, “ An Unequal Cluster-Based Routing Protocol in Wireless Sensor Network” Wireless Networks, Springer, Vol. 15, No. 2, 2009, pp. 193-207.

[12]. J. Ma, W. Lou, Y. Wu, M. Li, G. Chen, "Energy Efficient TDMA Sleep Scheduling in TDMA in wireless sensor networks,” IEEE Infocom, 2009, pp. 630-638

[13]. Jian Peng, Tang Liu, Hongyou Li, Bing Guo "EnergyEfficient Prediction Clustering Algorithm multilevel Heterogeneous Wireless Sensor Network” International Journal of Distributed Sensor Networks, Hindawi, Vol.2013, 2013 pp.1-7

[14]. G. Xing, X. Wang, Y. Zhang, C. Lu, R. Pless, C. Gill, "Integrated Coverage and Connectivity Configuration for Energy Conservation in Sensor Networks", ACM Transaction on Sensor Networks, Vol. 1, No. 1, 2005, pp. 36-72. 